lichkeit mit entsprechenden Konsequenzen für die Ausstattung und - last but not least - den Personalschlüssel, verstärkt die Tendenz, ärztliches oder pflegerisches Handeln als ,gefahrgeneigte Tätigkeiten ' betrachten zu müssen.“

\section{Knackpunkt Dokumentation}

Obwohl diese Entwicklung nicht ohne Folgen für die Qualität der Versorgung bleibe, könne das deutsche Haftungsrecht mit dem Verbot des Ausforschungsbeweises nur begrenzt eingreifen. Der Grund: Organisationsdefizite im Klinikbetrieb spiegeln sich nur selten in der individuellen Behandlungsdokumentation des Patienten wider, erläuterte Gaidzik. „Mängel in der personellen Besetzung, Abteilungskoordination und nicht zuletzt -kommunikation oder auch in der Organisation der Rufbereitschaft werden in aller Regel nur per Zufall zu Tage treten." Der Patient werde kaum in Erfahrung bringen können, ob ein Behandlungsfehler das Resultat eines Konzentrationsmangels des übermüdeten Assistenzarztes oder seiner unguten Erfahrungen mit einem mehrfach aus dem Schlaf gerissenen Facharztes im Hintergrunddienst ist. „Um hier das Haftungs- recht wirkungsvoll als Instrument einzusetzen, bedürfte es neuer Wege im Prozessrecht", sagte Gaidzik.

Für die Zukunft hält es der Medizinrechtler auch für denkbar, dass sich die Gutachterkommissionen und Schlichtungsstellen im Bereich der Mediation engagieren könnten. Grundsätzlich entlastet die Arbeit der Kommissionen nach Einschätzung von Gaidzik nicht nur die Gerichte. Sie ist auch in der Lage, die dort durch den fehlenden medizinischen Diskurs bestehenden Defizite auszugleichen, „mit vielleicht sogar weit größerem Potenzial in der Zukunft“.

Die nordrheinische Gutachterkommission hat in den 40 Jahren ihres Bestehens mehr als 50.000 Anträge bearbeitet. Dabei haben die Ärzte und Juristen bei etwa einem Drittel einen Behandlungsfehler festgestellt. „Wiederholte Auswertungen der Ergebnisse haben nachgewiesen, dass es der Gutachterkommission in bis $\mathrm{zu}$ 90\% der Begutachtungsfälle gelingt, den Haftungsstreit zwischen Patienten und Ärzten durch das für die Beteiligten kostenfreie Begutachtungsverfahren beizulegen“, lobte der Vizepräsident der Ärztekammer Nordrhein Bernd Zimmer.

Ilse Schlingensiepen

\title{
Anwälte dürfen mit Ärzten kooperieren
}

\section{Partnerschaftsgesellschaften von Ärzten und Apothekern mit Rechtsanwälten sind künftig erlaubt. Geklärt hat das jetzt das Bundesverfassungsgericht.}

U m eine umfassende, fachlich-juristische Beratung anbieten zu können, tun sich Rechtsanwälte häufig mit spezialisierten Kollegen, aber auch mit anderen Berufsgruppen zusammen. Zulässig ist dies laut Bundesrechtsanwaltsordnung (BRAO) allerdings nur mit Steuerberatern und -bevollmächtigten, Wirtschaftsprüfern sowie vereidigten Buchprüfern. Die Liste gilt als abschließend. Im Umkehrschluss waren Partnerschaftsgesellschaften mit anderen Berufen, darunter etwa Ärzten und Apothekern, daher bisher verboten. Dennoch hatten ein Rechtsanwalt und eine Ärztin und Apothekerin im Raum Würzburg eine gemeinsame Gesellschaft gegründet. Tätigkeit sollten Gutachten und Be-

ratung im Bereich des Arzt- und Apothekenrechts sein. Patienten behandeln wollte die Ärztin nicht. Das Amtsgericht Würzburg lehnte die Eintragung in das Partnerschaftsregister unter Hinweis auf die BRAO ab. Dem folgte auch das Oberlandesgericht Bamberg. In dritter Ins$\tan z$ hatte der Bundesgerichtshof jedoch verfassungsrechtliche Bedenken. Er legte die BRAO-Vorschrift daher dem Bundesverfassungsgericht zur Prüfung vor. Das schloss sich den Argumenten des Bundesgerichtshofs an. Die Regelung sei mit dem Grundrecht der Berufsfreiheit unvereinbar, befanden die Verfassungsrichter in ihrem jetzt schriftlich veröffentlichten Beschluss unter Aktenzeichen 1 BvL 6/13. Martin Wortmann

\section{Übersetzungshilfen gegen Sprachbarrieren}

Ärzte, die viele Flüchtlinge behandeln oder ihre Praxis in Regionen mit einem hohen Ausländeranteil führen, können für die Behandlung und zur Information ihrer Patienten auf umfangreiche Materialien zur Überwindung von Sprachbarrieren zurückgreifen. Die Praxishilfen werden von Landesärztekammern, direkt von der KBV oder auch von Verlagen bereitgestellt. Die KBV hat die unterschiedlichen Angebote auf ihrer Website zusammengestellt und verlinkt: www. kbv.de/html/1150_20914.php

Hauke Gerlof

\section{Ärztlicher Notdienst bei vielen Patienten unbekannt}

Der ambulante Bereitschaftsdienst, der durch die KV organisiert wird, leidet offenbar an einem Informationsdefizit seitens der Patienten. Das legt eine Befragung in zwei Berliner Notfallaufnahmen in Krankenhäusern nahe. $89 \%$ der Patienten, die abends oder nachts die Notfallaufnahme aufsuchten, hatten demnach den ambulanten Bereitschaftsdienst zuvor nicht kontaktiert. $55 \%$ kannten das Angebot nicht, 35\% fürchteten, dort lange warten zu müssen. Rund 7.000 Patienten hatten im Mai und Juni 2015 die Rettungsstellen von sich aus aufgesucht, knapp 2.000 nahmen an der Befragung teil.

Hintergrund der Befragung: Viele Krankenhäuser klagen über eine wachsende direkte Inanspruchnahme der Notaufnahmen durch die Patienten. Im Krankenhausstrukturgesetz hat der Gesetzgeber daher die Einrichtung von Portalpraxen an Kliniken zur Soll-Vorschrift erhoben.

Besonders in Berlin gibt es wegen der direkten Konsultuation von Notaufnahmen durch Patienten sogar während der normalen Öffnungszeiten seit einigen Monaten Streit zwischen der KV und den Krankenhäusern, der bis zum Bundesgesundheitsministerium eskaliert worden ist. Die KV versucht dort, durch Vorgaben Anreize zu setzen, dass die Krankenhäuser Patienten in der Notaufnahme bei Bagatellerkrankungen gar nicht erst annehmen. In anderen Regionen, etwa in Hessen, versucht die KV, Bereitschaftsdienstpraxen möglichst in der Nähe oder auf dem Gelände von Krankenhäusern anzusiedeln, um Patienten besser steuern zu können.

Susanne Werner und Hauke Gerlof 